\title{
Computational Situation Theory
}

\author{
Erkan Tin and Varol Akman \\ Department of Computer Engineering and Information Science \\ Faculty of Engineering \\ Bilkent University \\ Bilkent, 06533 Ankara, Turkey \\ tin@bilkent.edu.tr and akman@bilkent.edu.tr
}

\begin{abstract}
Situation theory has been developed over the last decade and various versions of the theory have been applied to a number of linguistic issues. However, not much work has been done in regard to its computational aspects. In this paper, we review the existing approaches towards 'computational situation theory' with considerable emphasis on our own research.
\end{abstract}

\section{Introduction}

Situation theory is an attempt to develop a mathematical theory of meaning which will clarify and resolve some tough problems in the study of language, information, logic, philosophy, and the mind [11]. It was first formulated in detail by Jon Barwise and John Perry in 1983 [12] and has matured over the last decade [25]. Various versions of the theory have been applied to a number of linguistic issues, resulting in what is commonly known as situation semantics $[7,8,10,24,31,33,35,58]$. The latter aims at the construction of a unified and mathematically rigorous theory of meaning, and the application of such a theory to natural languages.

Mathematical and logical issues that arise within situation theory and situation semantics have been explored in numerous works $[8,10,12,24,25,33]$. In the past, the development of a mathematical situation theory has been held back by a lack of availability of appropriate technical tools. But by now, the theory has assembled its mathematical foundations based on intuitions basically coming from set theory and logic $[1,8,24,26]$. With a remarkably original view of information (which is fully adapted by situation theory) [28, 29], a 'logic,' based not on truth but on information, is being developed [25]. This logic ${ }^{1}$ will probably be an extension of first-order logic [5] rather than being an alternative to it.

Individuals, properties, relations, spatio-temporal locations, and situations are basic constructs of situation theory. The world is viewed as a collection of objects, sets of objects, properties, and relations. Infons ('unit' facts) [26] are discrete items of information and situations are first-class objects which describe parts of the real world. Information flow is made possible by a network of abstract 'links' between high-order uniformities, viz. situation types. One of the distinguishing characteristics of situation theory vis-à-vis another influential semantic and logical tradition [27] is that

\footnotetext{
${ }^{1}$ According to The Advanced Learner's Dictionary of Current English (by A. S. Hornby, E. V. Gatenby, and H. Wakefield, London, U.K.: Oxford University Press, 1958), logic is the science or art of reasoning, proof, and clear thinking. Thus, the commonly accepted equation logic $=$ first-order logic is highly suspect. (Cf. [6] for an extended argument on this.)
}

information content is context-dependent (where a context is a situation).

All these features may be cast in a rich formalism for a computational framework based on situation theory. Yet, there have been few attempts to investigate this $[17,33,40,46,49$, $52,59]$. Questions of what it means to do computation with situations and what aspects of the theory makes this suitable as a novel programming paradigm have not been fully answered in the literature.

Existing approaches towards a computational account of situation theory unfortunately incorporated only some of its original features $[15,16,17,33,48,49,52]$; the remaining features were omitted for the sake of achieving particular goals. This has caused conceptual and philosophical divergence from the ontology of the original theory-a dangerous and unwanted side effect. Some recent studies $[59,60,61]$ have tried to avoid this pitfall by simply sticking to the essentials of the theory and adopting the ontological features which were originally put forward by Barwise and Perry in [12] and clarified by Devlin in [25].

The remaining parts of this paper are structured as follows. Situation theory and situation semantics are reviewed in Section 2. Section 3 emphasizes the role of situation theory in natural language semantics. An argument as to why situations should be used in natural language processing and knowledge representation for semantic interpretation and reasoning is made in Section 4. In Section 5, computational aspects of the theory is discussed and existing approaches are reviewed, with some emphasis on our own work. Finally, Section 6 presents our concluding remarks.

\section{Situation Theory and Situation Semantics}

Situation theory is a mathematical theory of meaning [25]. According to the theory, individuals, properties, relations, spatio-temporal locations, and situations are the basic ingredients. The world is viewed as a collection of objects, sets of objects, properties, and relations.

Individuals are conceived as invariants; having properties and standing in relations, they persist in time and space. $O b$ jects are the parts of individuals. (Words are also objects, i.e., invariants across utterances.) All individuals, including spatio-temporal locations, have properties (like being fragile or red) and stand in relations to one another (like being earlier, being under).

A sequence such as $\left\langle r, x_{1}, \ldots, x_{n}\right\rangle$ where $r$ is an $n$-ary relation over the individuals $x_{1}, \ldots, x_{n}$ is called a constituent sequence. Suppose Alice was eating ice cream yesterday at home and she is also eating ice cream now at home. Both of these situations share the same constituent (eats, Alice, ice cream). These two events, occurring at the same location but at different times, have the same situation type $s$ (cf. [4] for the origin of this idea). Situation types are partial functions 
from relations and objects to the truth values 0 and 1 (a.k.a. polarity). The situation type $s$, in our example, assigns 1 to the constituent sequence $\langle$ eats, Alice, ice cream):

In $s:$ eats, Alice, ice cream; 1.

Thus, 〈eats, Alice, ice cream; 1$\rangle \in s$.

Actually, situation types can be more general. For example, a situation type in which someone is eating something at home 'contains' the situation in which Alice is eating ice cream at home. Suppose Alice is not present in the room where this paper is being written. Then, "Alice is eating ice cream" is not part of our situation $s$ and hence gets no truth value in $s$. This is unlike the case in say, logic programming, where the quoted sentence would get the truth value false $(0)$. (This is due to the closed world assumption, CWA.) Thus, situation theory allows partiality in a strong sense [33].

Situations in which a sequence is assigned both truth values are called incoherent. For instance, a situation $s^{\prime}$ is incoherent if $\langle$ has, Alice, $A \odot ; 0\rangle \in s^{\prime}$ and $\langle$ has, Alice, $A \varnothing ; 1\rangle \in s^{\prime}$. This is a situation in which Alice has the $A O$ and she does not have the $A \varnothing$ in a card game. There cannot be a real situation $s^{\prime}$ validating this. Nevertheless, the constituent sequence $\langle$ has, Alice, $A \varnothing\rangle$ may be assigned these truth values for spatio-temporally distinct situation types (say, $s^{\prime}$ and $s^{\prime \prime}$ ).

Situation types are, however, independent of locations. A location and a situation type mold a state of affairs which in fact is a static situation. In order to keep track of change, courses of events are used. A course of events is a partial function from locations to situation types and may contain information about events at more than one location. The course of events $e$ that Alice is eating ice cream at location $l_{1}$ (say 11:00 a.m., at home) and is sleeping at a temporally succeeding location $l_{2}$ (say 12:15 p.m., at home) is represented as follows:

$$
\begin{aligned}
& \text { In } e \text {, at } l_{1}: \text { eats, Alice, ice cream; } 1 \text {, } \\
& \text { at } l_{2}: \text { sleeps, Alice; } 1, \\
& l_{1}<l_{2} \text { and } l_{1} @ l_{2} .
\end{aligned}
$$

Spatio-temporal locations are allowed to stand in relation with each other in different ways: $l_{1}$ temporally precedes $l_{2}$ $\left(l_{1}<l_{2}\right), l_{1}$ temporally overlaps $l_{2}\left(l_{1} \circ l_{2}\right), l_{1}$ spatially overlaps $l_{2}\left(l_{1} @ l_{2}\right), l_{1}$ is temporally included in $l_{2}\left(l_{1} \subseteq_{t} l_{2}\right), l_{1}$ is spatially included in $l_{2}\left(l_{1} \subseteq_{s} l_{2}\right)$, and $l_{1}$ is spatio-temporally included in $l_{2}\left(l_{1} \subseteq l_{2}\right)$.

Permitting partiality has the advantage of distinguishing between logically equivalent statements. For example. the statements "Bob is angry" and "Bob is angry and Bob is shouting or Bob is not shouting" are logically equivalent in the classical sense [5]. In situation semantics, these two sentences will not have the same interpretation. A course of events $e$ describing the situation in which Bob is only angry will not contain any sequence about Bob's shouting, i.e., $e$ will be 'silent' on Bob's shouting. However, another course of

\footnotetext{
${ }^{2}$ Some utterances are about different situation types 'meeting' in one. Consider the utterance "Alice did not eat ice cream because she was ill." The courses of events may be formulated as follows:

In $e_{2}$, at $l_{2}$ : because, $e_{0}, e_{1} ; 1$, where in $e_{1}$, at $l_{1}:$ is, Alice, ill; 1 , in $e_{0}$, at $l_{0}$ : eats, Alice, ice cream; 0 , $l_{0} \circ l_{1}, l_{0} \subseteq l_{2}$, and $l_{1} \subseteq l_{2}$.
}

events $e^{\prime}$ describing Bob's being angry and either his shouting or not shouting will contain a sequence about Bob's shouting.

Situation semantics uses statements to classify real situations by the claims statements make. Claims are represented by coherent courses of events. These courses of events classify the real situations which validate them; a real situation $s$ validates a course of events $e$ in case the following holds: if $\left\langle r, x_{1}, \ldots, x_{n} ; 1\right\rangle \in e_{l}$ (or $\left\langle r, x_{1}, \ldots, x_{n} ; 0\right\rangle \in e_{l}$ ), then in $s$, the objects $x_{1}, \ldots, x_{n}$ stand (or do not stand) in the relation $r$ at $l$. A course of events $e$ at a location $l, e_{l}$, is also called a situation type. For example, assume the existence of a real situation in which Bob is really angry at $l$. A coherent course of events $e$ making the claim "Bob is angry" at $l$ is validated by this real situation.

According to situation theory, meanings of expressions reside in systematic relations between different types of situations. They can be identified with relations on discourse situations $d$, (speaker) connections $c$, the utterance $\varphi$ itself, and the described situation $e$. Some public facts about $\varphi$ (such as its speaker and time of utterance) are determined by the discourse situations [53]. The ties of the mental states of the speaker and the hearer with the world constitute $c$ [37].

A discourse situation involves the expression uttered, its speaker, the spatio-temporal location of the utterance, and the addressee(s). Each of these defines a linguistic role (the role of the speaker, the role of the addressee, etc.) and we have a discourse event. For example, if the indeterminates $a, b, \alpha$, and $l$ denote the speaker, the addressee, the utterance, and the location of the utterance, respectively, then a discourse event $D$ is given as:

$$
\begin{aligned}
D:=\text { at } l: & \text { speaking, } a ; 1, \\
& \text { addressing, } a, b ; 1, \\
& \text { saying, } a, \alpha ; 1 .
\end{aligned}
$$

Using a name or a pronoun, the speaker refers to an individual. $^{3}$ A situation $s$ in which the referring role is uniquely filled is called a referring (anchoring) situation. If in $s$ the speaker uses a noun phrase $\nu$ to refer to a unique individual, this individual is called the referent of $\nu{ }^{4}$

Tense markers of tensed verb phrases can also refer to individuals, e.g., spatio-temporal locations. Therefore, an anchoring situation $s$ can be seen as a partial function from the referring words $\nu_{i}$ to their referents $s\left(\nu_{i}\right)$. This function is the speaker's connections for a particular utterance [53].

The utterance of an expression $\varphi$ 'constrains' the world in a certain way, depending on how the roles for discourse situations, connections, and described situation are occupied. For example, "I am crying" describes a three-place relation [I am crying】 on the utterance situation (the discourse situation and the connections) $u$ and the described situation $e$. This relation defines a meaning relation written in the following form:

\section{$d, c \llbracket$ I am crying $\rrbracket e$.}

Given a discourse situation $d$, connections $c$, and a course of events $e$, this relation holds just in case there is a location $l_{d}$

${ }^{3}$ A name directly refers to an individual, independent of whether the individual is imaginary or real. A pronoun can either refer to an individual deictically or else it may be used indirectly by co-referring with a noun phrase.

${ }^{4}$ Obviously, the speaker may not refer to anything at all. In this case, the role of the referent is left empty. 
and a speaker $a_{d}$ such that $a_{d}$ is speaking at $l_{d}$, and in $e, a_{d}$ is crying at $l_{d}$.

In interpreting the utterance of an expression $\varphi$ in a context $u$, there is a flow of information, partly from the linguistic form encoded in $\varphi$ and partly from contextual factors provided by the utterance situation $u$. These are combined to form a set of constraints on the described situation $e$ which is not uniquely determined: given $u$ and an utterance of $\varphi$ in $u$, there will be several situations $e$ that satisfy the constraints imposed. The meaning of an utterance of $\varphi$ and hence its interpretation are influenced by other factors such as stress, modality, and intonation [33]. However, the situation in which $\varphi$ is uttered and the situation $e$ described by this utterance seem to play the most influential roles. For this reason, the meaning of an utterance is essentially taken to be a relation defined over $\varphi, u(d, c)$, and $e$. This approach towards identifying linguistic meaning is essentially what Barwise and Perry call the Relation Theory of Meaning $[12,13]$.

The constituent expressions of $\varphi$ do not describe a situation when uttered in isolation. Uttering a verb phrase in isolation, for example, does not describe a situation $e$. Other parts of the utterance (of which this verb phrase is a part) must systematically contribute to the description of $e$ by providing elements such as an individual or a location. For example, the situational elements for the utterance of the tenseless verb phrase 'running' provide a spatio-temporal location for the act of running and the individual who is to run. For the tensed verb phrase 'is running,' an individual must be provided. The situational elements prepare the setting $\sigma$ for an utterance. The elements provided by $\sigma$ can be any individual, including spatio-temporal locations. The meaning of $\varphi$ is a relation defined not only over $d, c$, and $e$, but also over $\sigma$.

\section{Situation Semantics as Natural Language Semantics}

Language is an integral part of our everyday experience. Some activities pertaining to language include talking, listening, reading, and writing. These activities are situated; they occur in situations and they are about situations [4]. What is common to these situated activities is that they convey information $[25,28,29,38]$. When uttered at different times by different speakers, a statement can convey different information to a hearer and hence can have different meanings. ${ }^{5}$

This information-based approach to the semantics of natural languages has resulted in what is known as situation semantics. Situation semantics makes simple assumptions about the way natural language works. Primary among them is the assumption that language is used to convey information about the world (the so-called external significance of language). ${ }^{6}$ Even when two sentences have the same inter-

${ }^{5}$ Consider the sentence "That really attracts me." Depending on the reference of the demonstrative, interpretation (and hence meaning) would change. For example, this sentence could be uttered by a boy referring to a cone of ice cream or by a cab driver referring to fast driving, meaning absolutely different things [37].

"For example, "Bob smashed his car yesterday" conveys the information that there is an individual named Bob, that he has a car, that he crashed it, that this event occurred in the past, and that he was the driver of the car at the spatiotemporal location of this unfortunate event. Thus, sentences describe situations in the world. These situations and the pretation, i.e., they describe the same situation, they can carry different information. ${ }^{7}$

Classical approaches to semantics underestimate the role played by context-dependence; they ignore pragmatic factors such as intentions and circumstances of the individuals involved in the communicative process $[4,37,38]$. But, indexicals, demonstratives, tenses, and other linguistic devices rely heavily on context for their interpretation and are fundamental to the way language conveys information [2]. Contextdependence is an essential hypothesis of situation semantics. A given sentence can be used over and over again in different situations to say different things (the so-called efficiency of language). Its interpretation, i.e., the class of situations described by the sentence, is therefore subordinate on the situation in which the sentence is used. This context-providing situation, discourse situation, is the speech situation, including the speaker, the addressee, the time and place of the utterance, and the expression uttered. Since speakers are always in different situations, having different causal connections to the world and different information, the information conveyed by an utterance will be relative to its speaker and hearer (the so-called perspectival relativitv of language) [12].

Besides discourse situations, the interpretation of an utterance depends on the speaker's connections with objects, properties, times and places, and on the speaker's ability to exploit information about one situation to obtain information about another. Therefore, context supports not only facts about speakers, addressees, etc. but also facts about the relations of discourse-participants to other contextually relevant situations such as resource situations. Resource situations are contextually available and provide entities for reference and quantification. Their use has been demonstrated in the theory of definite descriptions of Barwise and Perry [12]. ${ }^{8}$

Another key assumption of situation semantics is the socalled productivity of language: we can use and understand expressions never before uttered [19]. Hence, given a finite vocabulary, we can form a potentially infinite list of meaningful expressions. The underlying mechanism for such an ability seems to be compositionality. ${ }^{9}$

objects in them have properties and stand in relations to each other at spatio-temporal locations.

${ }^{7}$ For example, "Bob went to the theater" and "The father of Carol went to the theater" both describe the same situation in which Bob (an individual) went to the theater, assuming that Bob is Carol's father. However, while the first sentence says that this individual is Bob, the second sentence conveys the information that Carol (another individual) has a father who went to the theater.

${ }^{8}$ Imagine, for example, that there are two card games going on, one across town from the other: Max is playing cards with Emily and Claire is playing cards with Dana. Suppose Bob watching the former game mistakes Emily for Claire, and utters the sentence "Claire has the three of clubs." According to the classical (Russelian) theories [32], if Claire indeed has 3 , this claim would be true since the definite noun phrases "Claire" and "the three of clubs" are used to pick out, among all the things in the world, the unique objects satisfying the properties of being an individual named Claire and being a 3 , respectively; the sentence would be considered to contain no explicit context-sensitive elements [10]. In contrast, situation semantics identifies these objects with respect to some limited situation-the resource situation exploited by Bob. The claim would then be wrong even if Claire had $3 \$$ across town. Thus, context is, in general, taken not to be a single situation, but a 'constellation' of related situations.

${ }^{9}$ The assumption that meaning of a larger linguistic unit 
Situation semantics closes another gap of traditional semantic approaches: the neglect of subject matter and partiality of information. In traditional semantics, statements which are true in the same models convey the same information [14]. Situation semantics takes the view that logically equivalent sentences need not have the same subject matter, they need not describe situations involving the same object and properties. The notion of partial situations (partial models) leads to a more fine-grained notion of information content and a stronger notion of logical consequence that does not lose track of the subject matter (and hence enhances the notion of relevance) [55].

The ambiguity of language is taken as another aspect of the efficiency of language. Natural language expressions may have more than one meaning. We have earlier noted that there are factors such as intonation, gesture, the place of an utterance, etc. which play a role in interpreting an utterance [33]. Instead of throwing away ambiguity and contextual elements, situation semantics tries to build up a full theory of linguistic meaning by initially isolating some of the relevant phenomena in a formal way and by exploring how the rest would help in achieving the goal [12].

According to situation semantics, we use meaningful expressions to convey information not only about the external world but also about our minds (the so-called mental significance of language). ${ }^{10}$ Situation semantics differs from other approaches in that we do not, in attitude reports, describe our mind directly (by referring to states of mind, ideas, senses, thoughts, etc.) but indirectly (by referring to situations that are external).

With these underlying assumptions and features, situation semantics provides a fundamental and appropriate framework for a realistic model-theoretic semantics of natural language [11]. Various versions of this theory have been applied to a number of linguistic issues (mainly) in English $[7,8,10,21,23,24,31,35,50]$. The ideas emerging from research in situation semantics have also been coalesced with well-developed linguistic theories such as lexical-functional grammar [54] and led to rigorous formalisms [33]. On the other hand, situation semantics has been compared to other influential mathematical approaches to the theory of meaning, viz. Montague Grammar [22, 27, 51] and Discourse Representation Theory (DRT) [42].

\section{Why Compute with Situations?}

We believe that a computational formulation of situation theory will generate interest among artificial intelligence and natural language processing researchers alike. The theory claims that its model theory is more amenable to a computationally tractable implementation than standard model theory (of predicate calculus) or the model theory of Montague

is a function of the meanings of its individual parts is called the principle of compositionality [11]. It can be considered as a reflection of the similar principle in logic $[5,18]$.

${ }^{10}$ Returning to a previous example, consider the sentence "A bear is coming this way" uttered by Bob. It can give us information about two different situations. The first one is the situation which we are located in. The second one is the situation which Bob believes. If we know that Bob is hallucinating, then we might learn the second situation, but not the first [12]. Focusing on the second situation, if we could not see any bear around, we would normally focus on Bob's belief situation.
Grammar. ${ }^{11}$ This is due to the fact that situation theory emphasizes partiality whereas standard model theory is clearly holistic.

From a natural language processing point of view, situation theory is interesting and relevant simply because the linguistic account of the theory (viz. situation semantics) handles various linguistic phenomena with a flexibility that surpasses other theoretical proposals. It seems that indexicals, demonstratives, referential uses of definite descriptions, deictic uses of pronouns, tense markers, names, etc. all have technical treatments in situation semantics that reach beyond available theoretical apparatus. For example, the proposed mechanisms, as reported in [35], for dealing with quantification and anaphoric connections ${ }^{12}$ in English sentences are all firmly grounded in situation semantics. The insistence of situation semantics on contextual interpretation makes the theory more compatible with speech act theory [53] and discourse pragmatics than other theories. ${ }^{13}$

With regard to interpretation, it should finally be remarked that there are other interesting approaches, e.g., Hobbs et al.'s 'interpretation as abduction' [39]. An abductive explanation is the most economical explanation coherent with the rest of what we know. According to Hobbs et al., to interpret a text is to prove abductively that it is coherent. ${ }^{14}$ Likewise, the process of interpreting sentences in discourse can be viewed as the process of giving the best explanation of why the sentences would be true. In the TACITUS project at SRI, Hobbs and his coworkers have developed a scheme for abductive inference that provides considerable advantage in the description of such interpretation processes.

While we do not regard abduction's philosophical foundation as sufficiently general and intuitive as that of situation semantics, it nonetheless gives a framework in which assorted tasks of linguistic processing can be formalized in a rather integrated fashion.

\section{Situations: A Computational Perspective}

Intelligent agents generally make their way in the world by being able to pick up certain information from a situation, process it, and react accordingly [25, 28, 29, 41]. Being in a (mental) situation, such an agent has information about

\footnotetext{
${ }^{11}$ Montague's intensional logic is particularly problematic in that the set of valid formulas are not recursively enumerable. Therefore, few natural language processing systems attempt to use it; the general inclination is to employ less expressive but more tractable knowledge representation formalisms.

${ }^{12}$ Gawron and Peters [35] focus on the semantics of pronominal anaphora and quantification. They argue that the ambiguities of sentences with pronouns can be resolved with an approach that represents anaphoric relations syntactically. This is achieved in a relational framework which considers anaphoric relations as relations between utterances in context.

${ }^{13}$ Kamp's DRT may safely be considered as the only serious competition in this regard [43]. However, it should be noted that there are currently research efforts towards providing an 'integrated' account of situation semantics and DRT, as witnessed by Barwise and Cooper's recent work [9].

${ }^{14}$ Similarly, it may be hypothesized (as Hobbs does) that faced with any situation (scene), we must prove abductively that it is a coherent situation. (Clearly, in the latter, part of what coherence means is clarifying why the situation exists.)
} 
situations it sees, believes in, hears about, etc. Alice, for example, upon hearing Bob's utterance "A bear is running towards you," would have the information, by relying on the utterance situation, that her friend is the utterer and that he is addressing her by the word "you." Moreover, by relying on the situation the utterance described, she would know that there is a bear around and it is running towards her.

Situations can be of the same type. Among the invariants across situations are not just objects and relations, but also aggregates of such. Having heard the warning above, Alice would realize that she is faced with a type of situation in which there is a bear and it is running. She would form a 'thought' over the running bears-an abstract object which carries the property of both being a bear and running-and on seeing the bear around, would individuate it.

Realization of some type of situation causes the agent to acquire more information about that situation as well as other situation types, and to act accordingly. Alice, upon seeing the bear around, would run away, being in possession of the previously acquired information that bears might be hazardous. She can obtain this information from the situation by means of some constraint-a certain relationship between bears and their fame as life-threatening creatures. Attunement to, or awareness of, that constraint is what enables her to acquire and use that information. ${ }^{15}$

An important phenomenon in situation theory is that of structured (nested) information [29]. Assuming the possession of prior information and/or awareness of other constraints, the acquisition by an agent of an item of information can also provide the agent with an additional item of information. On seeing a square, for example, one gains the information that the figure is a rectangle, and that it is a parallelogram, and that its internal angles are 90 degrees, and so on.

Reaping information from a situation is not the only way an agent processes information. It can also act in accordance of the obtained information to change the environment. Creating new situations to arrive at new information and conveying information it already had to other agents are the primary functions of its activities. Having the information that there is a bear around, Alice would run away, being attuned to the constraint that the best way to avoid danger in such situations is to keep away from the bear. Or, having realized that she cannot move, she would yell for help, being aware of the constraint that calling people in such situations might work.

In short, an intelligent agent has the ability to acquire information about situations, obtain new information about them (by being attuned to assorted constraints), and act accordingly to alter its environment. All these are ways of processing information about situations. An information processing environment for such an agent should have the following properties:

\footnotetext{
${ }^{15}$ To rehearse another classical example due to Barwise [25], a tree stump in a forest conveys various types of information to say, a hunter. If he is aware of the relationship between the number of rings in a tree trunk and the age of the tree, the stump will provide him the age of the tree when it was felled. If the hunter is able to recognize various kinds of bark, the stump can provide the information as to what type of tree it was, its probable height, shape, etc. To someone else the same tree stump could yield information about the weather the night before, the kinds of insects that live in the vicinity, and so on.
}

- Partitioning of information into situations.

- Parametrization of objects to give a proper treatment of abstraction over individuals, situations, etc.

- Structuring of situations in such a way that they allow nested information.

- Access to information partitioned in this way.

- Access to information in one situation from another situation connected to the former via some relation.

- Constraint satisfaction to control flow of information within a situation and between situations.

These properties would naturally define the underlying mechanisms for a situation-theoretic computational environment. But what constructs are provided by situation theory to build such an environment?

In situation theory, infons are the basic units of information [26]. Abstraction can be captured in a primitive level by allowing parameters in infons. Parameters are generalizations over classes of non-parametric objects (e.g., individuals, spatial locations). Parameters of a parametric object can be associated with objects which, if they were to replace the parameters, would yield one of the objects in the class that parametric object abstracts over. The parametric objects actually define types of objects in that class. Hence, letting parameters in infons results in parametric infons. For example, $\langle$ see, $X$, Alice; 1 ) and (see, $X, Y ; 1\rangle$ are parametric infons where $X$ and $Y$ are parameters over individuals. These infons are said to be parametric on the first, and first and second argument roles of the relation see, respectively. Parametric infons can also be allowed to be indetermined with respect to relation and polarity, e.g., $\langle R, X, Y ; I\rangle$ where $R$ and $I$ are parameters over relations and polarity, respectively. Parameter-free infons are the basic items of information about the world (i.e., 'facts') while parametric infons are the basic units that are utilized in a computational treatment of information flow.

To construct a computational model of situation theory, it is convenient to have available abstract analogs of objects. As noted above, by using parameters we can have parametric objects, including parametric situations, parametric individuals, etc. This yields a rich set of data types. Abstract situations can be viewed as models of real situations. They are set-theoretic entities that have only some of the features of real situations, but are amenable to computation. We define abstract situations as structures consisting of a set of parametric infons. Information can be partitioned into situations by defining a hierarchy between situations. A situation can be larger, having other situations as its subparts. (For example, an utterance situation for a sentence consists of the utterance situations for each word forming the sentence.) Being in this larger situation gives the ability of having information about its subsituations. The part-of relation ${ }^{16}$ of situation theory can be used to build such hierarchies among abstract situations and the notion of nested information can be accommodated.

Being in a situation, one can derive information about other situations connected to it in some way. For example, from an utterance situation it is possible to obtain information about the situation it describes. Accessing information both via a

${ }^{16}$ The part-of relation is reflexive, anti-symmetric, and transitive. Hence, it provides a partial-ordering of the situations [25, p. 72]. 
hierarchy of situations and explicit relationships among them requires a computational mechanism. This mechanism will put information about situation types related in some way into comfortable reach of the agent and can be made possible by a proper implementation of the supports relation, $\models$, of situation theory ( $\mathrm{cf}$. the 'extensionality principle' in [25, p. 72].) Given an infon $\sigma$ and a situation $s$, this relation holds if $\sigma$ is made true by $s$, i.e., $s \models \sigma$.

Barwise and Perry identify three forms of constraints [12]. Necessary constraints are those by which one can define or name things, e.g., "Every dog is a mammal." Nomic constraints are patterns that are usually called natural laws, e.g., "Blocks drop if not supported." Conventional constraints are those arising out of explicit or implicit conventions that hold within a community of living beings, e.g., "The first day of the month is the pay day." They are neither nomic nor necessary, i.e., they can be violated. All types of constraints can be conditional and unconditional. Conditional constraints can be applied to situations that fulfill some condition while unconditional constraints can be applied to all situations.

Constraints enable one situation to provide information about another and serve as links. (They actually link the types of situations.) Constraints can be used as inference rules in a computational system. When viewed as a backward-chaining rule, a constraint can provide a channel for information flow between types of situations, from the antecedent to the consequent. This means that such a constraint behaves as a 'definition' for its consequent part [57]. Another way of viewing a constraint is as a forwardchaining rule. This approach enables an agent to alter its environment. ${ }^{17}$

\subsection{Approaches to 'Computational Situation Theory'}

\subsubsection{PROSIT}

PROSIT (PROgramming in SItuation Theory) is the pioneering work in this direction. PROSIT is a situation-theoretic programming language developed by Nakashima et al. [49]. It has been implemented in Common Lisp [56].

PROSIT is tailored more for knowledge representation in general than for natural language processing. One can define situation structures and assert knowledge in particular situations. It is also possible to define relations between situations in the form of constraints. PROSIT's computational power is due to an ability to draw inferences via rules of inference which are actually constraints of some type. There is an inference engine similar to a Prolog interpreter [57]. PROSIT offers a treatment of partial objects, such as situations and parameters. It can deal with self-referential expressions [10].

One can assert facts that a situation will support. For example, if S1 supports the fact that Bob is a young person, this can be defined in the current situation $S$ as:

$$
\mathrm{S}:(\vDash \mathrm{S} 1 \text { (young "Bob")) }
$$

Note that the syntax is similar to that of Lisp and the fact is in the form of a predicate. The supports relation, $\models$, is

\footnotetext{
${ }^{17}$ For instance, being aware of a man ringing the door bell, Alice utters the sentence "A man is at the door." This in turn results in Carol's (another agent's) opening the door. Or it introduces into the discourse a noun phrase for pronominalization in the subsequent discourse, e.g., Bob's question: "Is he the mailman?"
}

situated so that whether a situation supports a fact depends on within which situation the query is made. Queries can be posed about one situation from another, but the results will depend on where the query is made.

There is no notion of situation type in PROSIT. For this reason, one cannot represent abstractions over situations and specify relations between them without having to create situations and assert facts to them.

PROSIT has a constraint mechanism. Constraints can be specified using either of the three relations $\Rightarrow, \Leftarrow$, and $\Leftrightarrow$. Constraints specified using $\Rightarrow$ (respectively, $\Leftrightarrow$ ) are forward (respectively, backward) chaining constraints; the ones using $\Leftrightarrow$ are both backward-and forward-chaining constraints. Backward chaining constraints are of the form $\left(\Leftarrow\right.$ head $_{\text {fact }}$ $\left.\ldots \mathrm{fact}_{n}\right)$. If all the facts are supported by the situation, then the head fact is supported by the same situation. Forward chaining constraints are of the form $\left(\Rightarrow\right.$ fact tail l $_{1}$. tail $_{n}$ ). If fact is asserted to the situation, then all the tail facts are asserted to the same situation. Backward chaining constraints are activated at query-time while forward-chaining constraints are activated at assertion-time. By default, all the tail facts of an activated forward-chaining constraint are asserted to the situation, which may in turn activate other forward-chaining constraints recursively.

For a constraint to be applicable to a situation, the situation must be declared to 'respect' the constraint. This is done by using the special relation respect. For example, to state that every man is human, one would write:

$$
\text { S: }(\text { respect } S 1(\Leftarrow(\text { human } * X)(\operatorname{man} * X))) \text {. }
$$

This states that $\mathrm{S} 1$ respects the stated constraint and is made with respect to $S$. ( ${ }^{*} X$ denotes a variable.) Since assertions are situated, a situation will or will not respect a constraint depending on where the query is made. If we assert that:

$$
\mathrm{S}:(\vDash \mathrm{F} 1(\operatorname{man} \text { "Bob")), }
$$

then PROSIT will answer yes to the query:

$$
\text { S? (= S1 (human "Bob")). }
$$

The question mark indicates that the expression on its right is a query expression for the situation on its left.

Constraints in PROSIT are about local facts within a situation rather than about situation types. That is, the interpretation of constraints does not allow direct specification of constraints between situations, but only between infons within situations. (Situation theory allows constraints between situation types.)

Situated constraints offer an elegant solution to the treatment of conditional constraints which apply in situations that obey some condition. For example, when Alice throws a basketball, she knows it will come down-a constraint to which she is attuned, but which would fail if she tried to play basketball in a space shuttle. This is actually achieved in PROSIT since information is specified in the constraint itself. Situating a constraint means that it may only apply to appropriate situations. This is a good strategy to achieve background conditions. However, it might be required that conditions set not only within the same situation, but also between various types of situations. Because constraints have to be situated in PROSIT, not all situations of the appropriate type will have a constraint to apply. 
PROSIT does not provide an adequate mechanism for specifying conventional constraints, i.e., constraints which can be violated. An example of this sort of constraint is the relation between the ringing of the bell and the end of class. It is not logically necessary that the ringing of the bell should mean the end of class.

Parameters, variables, and constants are used for representing entities in PROSIT. Variables, rather than parameters, are used to identify the indeterminates in a constraint. $\mathrm{Pa}$ rameters might be used to refer to unknown objects in a constraint. Variables have a limited scope; they are local to the constraint in which they appear. Parameters, on the other hand, have global scope throughout the whole description. Variables match any expression in the language and parameters be can equated to any constant or parameter. That is, the concept of appropriateness conditions is not exploited in PROSIT. Appropriateness conditions, in fact, specify restrictions on the types of arguments a relation can take [25, p. 115]. It is more useful to have parameters that range over various classes rather than to work with parameters ranging over all objects. Such particularized parameters are known as restricted parameters $[25$, p. 53].

Some treatment of parameters is given in PROSIT with respect to anchoring. Given a parameter of some type (individual, situation, etc.), an anchor is a function which assigns an object of the same type to the parameter [25, pp. 52-63]. Hence, parameters work by placing restrictions on anchors. There is no appropriate anchoring mechanism in PROSIT since parameters are not typed.

PROSIT has been used to show how problems involving cooperation of multiple agents can be solved, especially by combining reasoning about situations. In [48], Nakashima et al. demonstrate how the Conway paradox ${ }^{18}$ can be solved. The agents involved in this problem use the common knowledge accumulated in a shared situation. This situation functions as a communication channel containing all information known to be commonly accessible. One agent's internal model of the other is represented by situations. Individual knowledge situation plus the shared situation help an agent to solve the problem; also cf. [30].

\subsubsection{ASTL}

Black's ASTL (A Situation Theoretic Language) is another programming language based on situation theory [17]. ASTL is aimed at natural language processing. One can define in ASTL constraints and rules of inference over the situations. An interpreter, a basic version of which is implemented in

${ }^{18}$ During a card game both Bob and Alice have an ace. Each of them thus knows that "Either Bob or Alice has an ace" is a fact. Now suppose Emily were to come along and ask them both whether they knew if the other one had an ace. They would answer "no," of course. And if Emily asked again (and again, ...) , they would still answer "no." But now suppose Emily said to them, "Look, at least one of you has an ace. Now do you know whether the other has an ace?" They would again both answer "no." But now something happens. Upon hearing Bob answer "no" Alice would reason as follows: "If Bob does not know I have an ace, having heard that one of us does, then it can only be because he has an ace." Bob would reason in the same way. So they both figure out that the other has an ace. Somehow, Emily's statement must have added some information. But how can that be, since Emily told them something that each of them already knew? This is known as the Conway paradox [8, pp. 201-220].
Common Lisp [56], passes over ASTL definitions and answers queries about the set of constraints and basic situations.

ASTL allows of individuals, relations, situations, parameters, and variables. These definitions form the basic terms of the language. Complex terms are in the form of i-terms (to be defined shortly), situation types, and situations. Situations can contain facts which have those situations as arguments. Sentences in ASTL are constructed from terms in the language and can be constraints, grammar rules, or word entries.

The complex term i-term is simply an infon ${ }^{19}$ $\left\langle r e l, a r g_{1}, \ldots, a r g_{n}, p o l\right\rangle$ where rel is a relation of arity $n, \arg _{i}$ is a term, and pol is either 0 or 1 . A situation type is given in the form $\left[\right.$ param $\mid$ cond $_{1} \ldots$ cond $\left._{n}\right]$ where cond $d_{i}$ has the form param $\models i$-term. If situation $\mathrm{S} 1$ supports the fact that Bob is a young person, this can be defined as:

$$
\text { S1: }[S \mid S \vDash\langle\text { young, bob, } 1\rangle] \text {. }
$$

The single colon indicates that $\mathrm{S} 1$ supports the situation type on its right-hand side. The supports relation in ASTL is global rather than situated. Consequently, query answering is independent of the situation in which the query is issued.

Constraints are actually backward-chaining constraints. Each constraint is of the form sit $t_{0}:$ type $_{0} \Leftarrow s_{i} t_{1}$ : type $_{1}, \ldots$, sit $_{n}$ : type ty $_{n}$, where sit $_{i}$ is a situation or a variable, and type $_{i}$ is a situation type. If each sit $_{i}, 1 \leq i \leq n$, supports the corresponding situation type, type $i$, then sit supports type $e_{0}$. For example, the constraint that every man is a human being can be written as follows:

$$
{ }^{*} \mathrm{~S}:\left[\mathrm{S} \mid \mathrm{S} \vDash\left\langle\text { human, }{ }^{*} \mathrm{X}, 1\right\rangle\right] \Leftarrow{ }^{*} \mathrm{~S}:\left[\mathrm{S} \mid \mathrm{S} \vDash\left\langle\operatorname{man},{ }^{*} \mathrm{X}, 1\right\rangle\right] \text {. }
$$

${ }^{*} \mathrm{~S},{ }^{*} \mathrm{X}$ are variables and $\mathrm{S}$ is a parameter. An interesting property of ASTL is that constraints are global, i.e., have a non-explicitly stated scope. Thus, a new situation of the appropriate type need not have a constraint explicitly added to it. For example, assume that S1, supporting the fact that Bob is a man, is asserted:

$$
\mathrm{S} 1:[\mathrm{S} \mid \mathrm{S} \vDash\langle\operatorname{man}, \mathrm{bob}, 1\rangle] \text {. }
$$

This together with the constraint above would give:

$$
\text { S1: }[S \mid S \vDash\langle\text { human, bob, 1)]. }
$$

Grammar rules are another form of constraints. An example grammar rule describing the utterance of a sentence consisting of a noun phrase and verb phrase can be defined as:

$$
\begin{aligned}
* \mathrm{~S}:[\mathrm{S} \mid \mathrm{S} & \models\langle\text { cat, } \mathrm{S}, \text { sentence, } 1\rangle] \rightarrow \\
& { }^{\mathrm{NPP}}:[\mathrm{S} \mid \mathrm{S} \vDash\langle\text { cat, } \mathrm{S}, \text { nounphrase, } 1\rangle], \\
& { }^{*} \mathrm{VP}:[\mathrm{S} \mid \mathrm{S} \vDash\langle\text { cat, } \mathrm{S}, \text { verbphrase, } 1\rangle]
\end{aligned}
$$

where cat denotes the category of the construct, and $\rightarrow$ indicates that this is a grammar rule. This rule can be read: "When there is a situation *NP of the given type and situation *VP of the given type, there is also a situation ${ }^{*} S$ of the given type."

Although one can define constraints between situations in ASTL, the notion of a background condition for constraints is not available. Similar to PROSIT, ASTL cares little about

\footnotetext{
${ }^{19}$ We use Black's notation almost verbatim rather than adapting it to the 'standard' notation of our paper.
} 


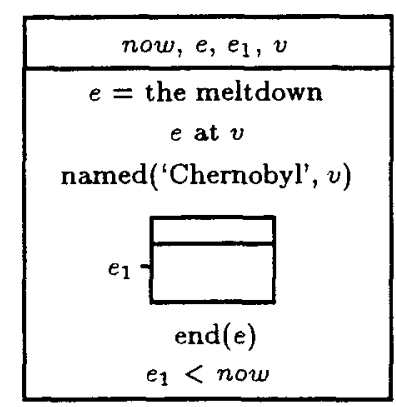

(a)

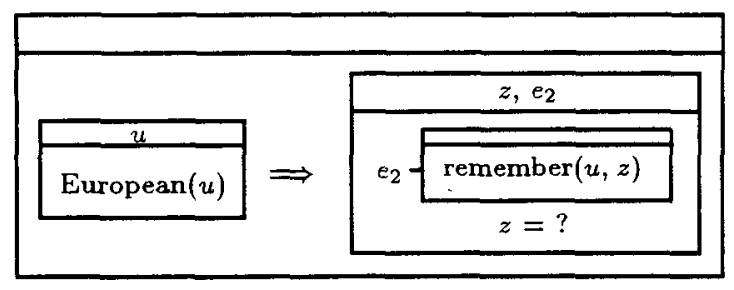

(b)

Figure 1: DRSs to model the discourse segment "The meltdown at Chernobyl has ended. Every European will remember it."

coherence within situations. This is left to the user's control. Accordingly, there is no mechanism in ASTL to specify constraints that can be violated.

Declaring situations to be of some type allows abstraction over situations to some degree. But, the actual means of abstraction over objects in situation theory, viz. parameters, do not carry much significance in ASTL.

As in PROSIT, variables in ASTL have scope only within the constraint they appear. They match any expression in the language unless they are declared to be of some specific situation type in the constraint. Hence, it is not possible to declare variables as well as parameters to be of other types such as individuals, relations, etc. Consequently, anchoring on parameters cannot be achieved appropriately in ASTL. Moreover, ASTL does not allow definition of appropriateness conditions for arguments of relations. 'Speaking' relation. for example, might require its speaker role to be filled by a human. Such a restriction could be defined only by using constraints of ASTL. However, this requires writing the restriction each time a new constraint about 'speaking' is to be added. Having appropriateness conditions as a built-in feature would be better.

ASTL does not have a mechanism to relate two situations so that one will support all the facts that the other does. This might be achieved via constraints, but there is no built-in structure between situations (as opposed to the hierarchy of situations in PROSIT).

The primary motivation underlying ASTL is to figure out a framework in which semantic theories such as situation semantics [8] and DRT [42] can be described and possibly

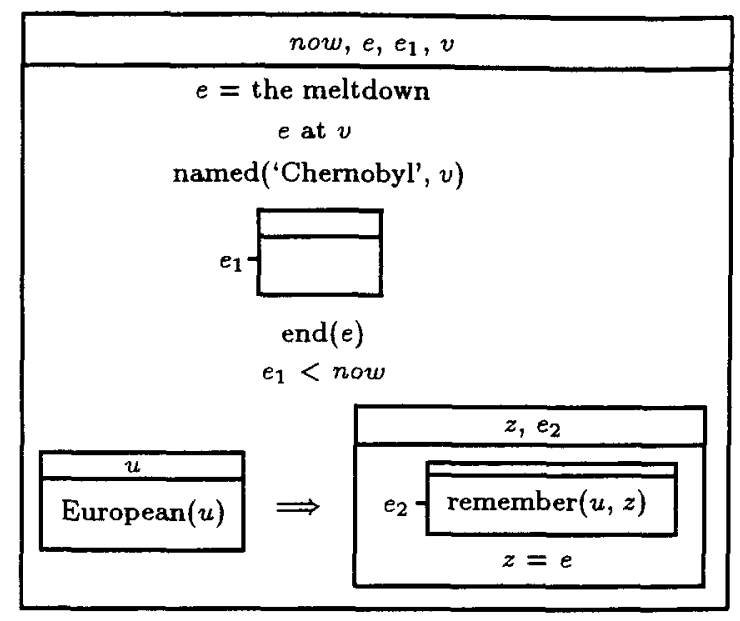

Figure 2: The unified DRS for Figure 1.

compared. ${ }^{20}$ (Such an attempt can be found in [16].) In DRT, a discourse representation structure (DRS) is defined at each stage in a discourse describing the current state of the analysis. A DRS consists of two parts: a set of domain markers (discourse referents), which can be bound to objects introduced into the current discourse, and a set of conditions on these markers. DRSs are typically drawn as boxes with the referents on the top window and conditions below. Figure 1 shows the DRSs for the sentences "The meltdown at Chernobyl has ended" and "Every European will remember it," respectively [3]. Individual discourse referents are denoted by now, $u, v$, and $z$ while event discourse referents are denoted by the letter $e$ (with or without subscripts). $e_{1}$ represents the whole event (ending of the meltdown at Chernobyl) described by the first sentence. Conditions are defined using basic predicates and logical operators. The DRS in Figure 1(b) is true if for every European, he can remember $z, z$ is a discourse referent identified by the anaphoric pronoun "it" and the rules of DRS construction require that "it" be matched with some previously introduced discourse referent. However, at the present stage there is no discourse referent with appropriate features. DRS construction can be completed by adding the discourse referents and the conditions introduced for the latter sentence to those declared for the former. Since the DRS for the first sentence contains a discourse referent with appropriate features, the second sentence can now be resolved. The 'unified' result is depicted in Figure 2.

\subsubsection{Situation Schemata}

Situation schemata have been introduced by Fenstad et al. [33] as a theoretical tool for extracting and displaying information relevant for semantic interpretation from linguistic form. A situation schema is in fact an attribute-value system which has a choice of primary attributes matching the primitives of situation semantics. The boundaries of situation schemata are however flexible and depending on the underlying theory of grammar, are susceptible to amendment. Hence, available linguistic insights can be freely exploited.

\footnotetext{
${ }^{20}$ For this reason, ASTL has specific built-in features for natural language processing. It is claimed that these features can be justified from a situation-theoretic view [17].
} 


$\operatorname{SIT} . \varphi\left[\begin{array}{ll}\text { REL } & r \\ \text { ARG.1 } & a \\ \text { ARG. } 2 & b \\ \text { LOC } & - \\ \text { POL } & i\end{array}\right] \quad\left[\begin{array}{lll}\text { IND } & \text { IND. } \alpha & \\ \text { COND } & {\left[\begin{array}{ll}\text { REL } & r \\ \text { ARG.1 } & {[]_{\alpha}} \\ \text { ARG.2 } & l o c_{d} \\ \text { POL } & i\end{array}\right]}\end{array}\right]$

(a)

(b)

Figure 3: (a) A prototype situation schema, (b) the general format of $\mathrm{LOC}$ in (a).

A simple sentence $\varphi$ has the situation schema shown in Figure $3(\mathrm{a})$. Here $r$ can be anchored to a relation, and $a$ and $b$ to objects; $i \in\{0,1\}$ gives the polarity. LOC is a function which anchors the described fact relative to a discourse situation $d, c$. LOC will have the general format in Figure 3(b). IND. $\alpha$ is an indeterminate for a location, $r$ denotes one of the basic structural relations on a relation set $R$, and $l o c_{0}$ is another location indeterminate. The notation [ $]_{\alpha}$ indicates repeated reference to the shared attribute value, IND. $\alpha$. A partial function $g$ anchors the location of SIT. $\varphi$, viz. SIT. $\varphi$.LOC, in the discourse situation $d, c$ if

$$
\begin{aligned}
& g\left(l o c_{0}\right)=l o c_{d} \text { and } \\
& c(r), g(\text { IND. } \alpha), l o c_{d} ; 1
\end{aligned}
$$

where $l o c_{d}$ is the discourse location and $c(r)$ is the relation on $R$ given by the speaker's connection $c$. The situation schema corresponding to "Alice saw the cat" is given in Figure 4.

Situation schemata can be adopted to various kinds of semantic interpretation. One could give some kind of operational interpretation in a suitable programming language, exploiting logical insights. But in its present form, situation schemata do not go further than being a complex attribute-value structure. They allow representation of situations within this structure, but does not use situation theory itself as a basis. Situations, locations, individuals, and relations constitute the basic domains of the structure. Constraints are declarative descriptions of the relationships holding between aspects of linguistic form and the semantic representation itself.

Theoretical issues in natural language semantics have been implemented on pilot systems employing situation schemata. The grammar described in [33], for example, has been fully implemented using a lexical-functional grammar system [34] and a fragment including prepositional phrases has been implemented using the DPATR format [20].

\subsubsection{EPILOG}

A recent addition to the small set of computational approaches to situation semantics is Episodic Logic (EL) introduced by Hwang and Schubert [40]. EL is theoretically inspired by Montague Semantics, with clear influences by situation semantics. EL is highly expressive and provides an easily computed first-order logical form for English (incorporating a DRT-like treatment). It covers English constructs ranging from sentences involving events, actions, attitudes to say, donkey sentences [36]. There is a straightforward

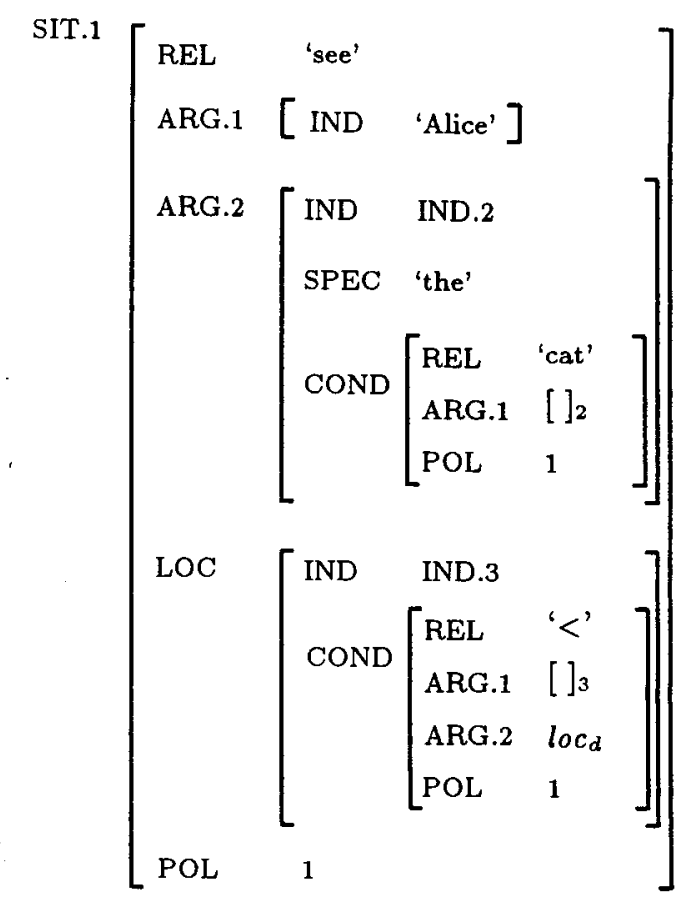

Figure 4: Situation schema for "Alice saw the cat."

transformation $^{21}$ of the initial indexical logical form to a nonindexical one.

Hwang and Schubert's deindexing algorithm uniformly handles tense and aspect, and removes context dependency by translating the context information into the logical form. Their EPILOG (the experimental computational system for episodic logic) is able to make some interesting inferences and answer questions based on logically represented simple narratives $^{22}$ (e.g., a children's story or a message processing application for commercial airplanes). This is a hybrid inference system incorporating efficient storage/access mechanisms, forward/backward chaining, and features to deal with taxonomies and temporal reasoning.

\subsubsection{BABY-SIT}

BABY-SIT is a computational medium based on situations, a prototype of which is currently being developed in $\mathrm{KEE}^{T M}$ (Knowledge Engineering Environment) [44]. The primary motivation underlying BABY-SIT is to facilitate the devel-

\footnotetext{
${ }^{21}$ Hwang and Schubert claim that such a translation is required because a situational logic must be nonindexical, i.e., it must not include atoms whose denotation depends on the utterance context. They justify this claim by stating that the facts derived by a system from natural language input may have been acquired in very different utterance contexts. Obviously, this shows that they depart markedly from situation semantics' well-known relation theory of meaning: while the meaning of a sentence is a relation between an utterance situation and a described situation in situation semantics, Hwang and Schubert : rgue that a nonindexical representation is essential.

${ }^{22}$ In fact, the adjective "episodic" is meant to suggest that in narrative texts, the focus is on time-bounded situations rather than atemporal ones.
} 


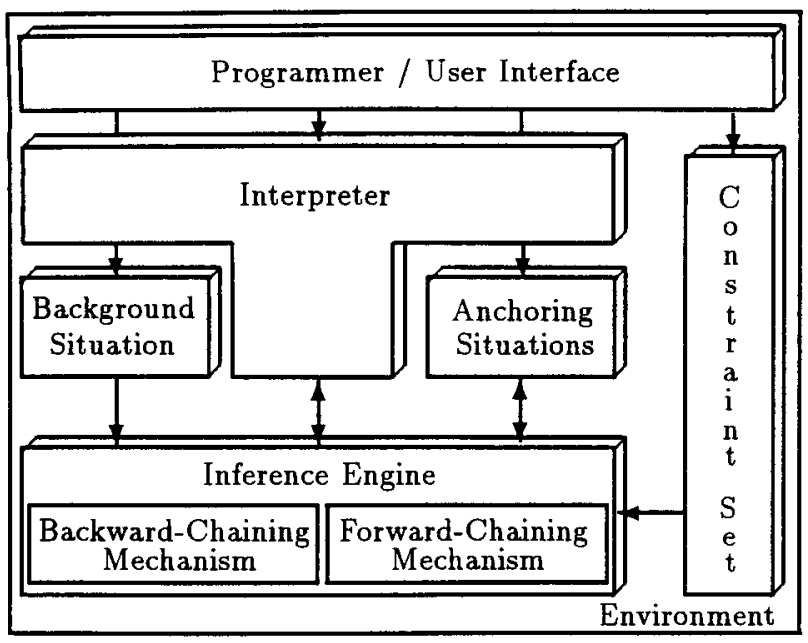

Figure 5: The architecture of BABY-SIT.

opment and testing of programs in domains ranging from linguistics to artificial intelligence in a unified framework built upon situation-theoretic constructs [60]. An interactive environment (cf. Figure 5) helps one to develop and test his program, observe its behavior vis-à-vis extra (or missing) information, make inference over the model, and issue queries [59].

The computational model underlying the current version of BABY-SIT consists of nine primitive domains: individuals, times, places, relations, polarities, parameters, infons, situations, and types. Each primitive domain carries its own internal structure. Individuals are unique atomic entities in the model which correspond to real objects in the world. Times are individuals of distinguished type, representing temporal locations and, similar to times, places are individuals which represent spatial locations. Various relations hold or fail to hold between objects. A relation has argument roles which must be occupied by appropriate objects. Infons are the discrete items of information of the form «rel, $\arg _{1}, \ldots, \arg _{n}$, pol $\gg$, where rel is a relation, $\arg _{i}, 1 \leq i \leq n$, is an object of the appropriate type for the $i$ th argument role, and pol is the polarity. Parameters are 'place holders' for objects in the model. They are used to refer to arbitrary objects of a given type. Types form higher-order uniformities for individuating or discriminating uniformities in the world.

(Abstract) situations are set-theoretic constructs, e.g., a set of parametric infons (comprising relations, parameters, and polarities). A parametric infon is the basic computational unit. If a situation $s$ supports an infon $\sigma$, this is denoted by $s \models \sigma$. Otherwise, it is written as $s \not \neq \sigma$. By defining a hierarchy between them, situations can be embedded via the special relation part-of. In this way, a situation $s$ can have information about another situation $s^{\prime}$ which is part of $s$, i.e., if $s^{\prime}=\sigma$, then $s \models \sigma$ for a given infon $\sigma$. A distinguished situation called background situation (denoted by $w$ ) contains infons which are inherited by all situation, i.e., the background situation is implicitly part of all situation structures in the environment and its infons hold in all situations. However, situations other than the background situation can contain infons that can vary from situation to situation. All situations are required to cohere. A situation can be either (spatially and/or temporally) located or unlocated. Time and place for a situation can be declared by time-of and place-of relations, respectively. A situation in the environment can only be realized if its parameters are anchored to objects in the real world. This is made possible by anchoring situations which allow parameters to be anchored to objects of appropriate types-individuals, situations, parameters, etc. But a parameter must be anchored to a unique object in an anchoring situation, i.e., it is anchored once in a given anchoring situation. On the other hand, more than one parameter may be anchored to the same object in an anchoring situation. Anchoring of a parameter can be done via the special relation anchor. Restrictions on parameters must be satisfied by the background situation. These restrictions assure anchoring of one parameter to an object having the same qualifications as the parameter. An anchoring situation has a functionality similar to that of the connections, $c$, mentioned in Section 2.

Assertion mode of BABY-SIT provides an interactive environment in which one can define objects and their types. There are nine basic types corresponding to nine primitive domains: $\sim$ IND (individuals), TIM (times), $\sim$ LOC (places), $\sim \mathrm{REL}$ (relations), $\sim \mathrm{POL}$ (polarities), INF (infons), $\sim \mathrm{PAR}$ (parameters), $\sim$ SIT (situations), and $\sim$ TYP (types). For instance, if $l$ is a place, then $l$ is of type $\sim$ LOC, and the infon $\ll$ of-type, $l, \sim$ LOC, $1 \gg$ is a fact in the background situation. Note that type of all types is $\sim$ TYP. For example, the infons $\ll$ of-type, $\sim \mathrm{LOC}, \sim \mathrm{TYP}, 1 \gg$ and $\ll$ of-type, $\sim \mathrm{TYP}, \sim \mathrm{TYP}$, $1 \gg$ are facts in the background situation by default. The syntax of the assertion mode (cf. [59]) is the same as in [25].

Suppose $b o b$ is an individual, see is a relation, and sit 1 is a situation. Then, these objects can be declared as:

$$
\begin{aligned}
& \text { I> bob: } \sim \mathrm{IND} \\
& \text { I> see: } \sim \mathrm{REL} \\
& \text { I> sit 1: } \sim \mathrm{SIT}
\end{aligned}
$$

The definition of relations includes the appropriateness conditions for their argument roles. Appropriateness conditions define the domains to which arguments of a relation belong. Each argument can be declared to be from one or more of the primitive domains above. Consider the seeing relation above. If we like it to have two arguments, the former being of type individual and the latter being of type situation, we can write:

$$
\mathrm{I}><\text { see } \mid \sim \mathrm{IND}, \sim \mathrm{SIT}>[1]
$$

The number in brackets at the right hand side of the expression indicate the minimum number of arguments that can be used with the seeing relation. Hence, $\ll s e e, b o b, 1 \gg$, for example, is considered to be a valid infon in the system.

In order for the parameters to be anchored to objects of the appropriate type, parameters must be declared to be from only one of the primitive domains. It is also possible to put restrictions on a parameter in the environment. Suppose we want to have a parameter $E$ denoting any individual that sees situation sit1. This can be done by asserting:

$$
\text { I> E }=\text { IND1 ^ «see, IND1, sit1, 1》 }
$$

IND1 is a default system parameter of type $\sim$ IND. E is considered as an object of type $\sim$ PAR such that if it is anchored to an object, say obj1, then obj1 must be of type $\sim$ IND and the background situation must support the infon $\ll s e e, o b j 1$, sit1, $1 \gg$.

Parametric types are also allowed in BABY-SIT. They can be formed by obtaining a type from a parameter. Parametric types are of the form $[P \mid s \models I]$ where $P$ is a parameter, $s$ is a situation (i.e., a grounding situation), and $I$ is a set of infons. The type of all situations that Bob sees can be defined in BABY-SIT as follows: 
I> $\operatorname{SITALL}=[\operatorname{SIT} 1 \mid w \models \ll s e e, b o b, \mathrm{SIT} 1,1 \gg]$

Hence, SITALL is seen as an object of type TYP in BABY-SIT and can be used as a type specifier for declaration of new objects in the environment. An object of type $\sim$ SITALL, say obj2, is an object of basic type SIT such that the background situation supports the infon $\ll s e e, b o b$, obj 2 , $1 \gg$.

Naming infons enables one to easily refer to them in expressions. For instance, the infon $\ll s e e, b o b, s i t 1,1 \gg$ can be named infon 1 by a sequence of assertions:

$$
\begin{aligned}
& \text { I> infon } 1: \sim \mathrm{INF} \\
& \text { 1 }>\text { infon } 1=\ll \text { see }, \text { bob }, \text { sit } 1,1 \gg
\end{aligned}
$$

In BABY-SIT, a situation browser enables one to create situations, browse them graphically, add or delete infons, and establish hierarchies among situations. Another way of achieving these, except graphical browsing infon deletion, is to use assertion mode. For example, the following sequence of assertions creates a situation sit 2 and then adds the infon $\ll s e e$, bob, sit $1,0 \gg$ into it:

$$
\begin{aligned}
& \text { I> sit2: } \sim \mathrm{SIT} \\
& \mathrm{I}>\text { sit2 } \models \ll s e e, b o b, \text { sit1, } 0 \gg
\end{aligned}
$$

Variables in BABY-SIT are only used in constraints and query expressions, and have scope only within the constraint or the query expression they appear. A variable can match any object appropriate for the place or the argument role it appears in. For example, given the declaration of the seeing relation above, variables ?S and ?X in the proposition ?SE $=\ll$ see, ?X, sit $1,1 \gg$ can only match objects of type $\sim \mathrm{SIT}$ and $\sim$ IND, respectively.

\section{A BABY-SIT constraint is of the form:}

$$
\begin{gathered}
\text { antecedent }_{1}, \ldots, \text { antecedent }_{n}\{\Leftarrow, \Rightarrow, \Leftrightarrow\} \\
\text { consequent }_{1}, \ldots, \text { consequent }_{m} .
\end{gathered}
$$

Each antecedent $t_{i}, 1 \leq i \leq n$, and each consequent $t_{j}$ $1 \leq j \leq m$, is of the form sit $\{\models, \not \models\} \ll r e l$, $\arg _{1}, \ldots$, argl, pol $\gg$ such that $r e l$ and each $a r g_{k}, 1 \leq k \leq l$, can either be an object of appropriate type or a variable.

Each constraint has an identifier associated with it and must belong to a group of constraints. For example, the following is a backward-chaining constraint named HUMAN-BEINGS-012 under the constraint group SPECIESPERSPECTIVE:

\section{SPECIES-PERSPECTIVE: \\ HUMAN-BEINGS-012:

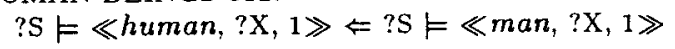

where ?S and ?X are variables. ?S can only be assigned an object of type $\sim$ SIT while ?X can have values of some type appropriate for the argument roles of the human and man relations. This constraint can apply in any situation. Hence, BABY-SIT constraints can be global. Constraints can also be situated. For example, HUMAN-BEINGS-012 constraint above can be rewritten to apply only in situation sit1:

$$
\text { sit } 1=\ll \text { k } \models_{\text {man, }} \mathrm{X}, 1 \gg \Leftarrow s i t 1 \models \ll \operatorname{man} . ? \mathrm{X}, 1 \gg \text {. }
$$

Conditional constraints of BABY-SIT come with a set of background conditions which must be satisfied for the constraint to apply. For example, to state that blocks drop if not supported, one can write:

\author{
NATURAL-LAW-PERSPECTIVE: \\ FALLING-BLOCK: \\ $? \mathrm{~S} 1 \vDash \ll$ block, ?X, 1》, \\ ?S1 $\vDash \ll$ supported, ?X, 0» $\Rightarrow$ ?S2 $\vDash \ll d r o p s, ? \mathrm{X}, 1 \gg$ \\ UNDER-CONDITIONS: \\ $w: \ll e x i s t s$, gravity, $1 \gg$.
}

Background conditions are, in fact, assumptions which are required to hold for constraints to be eligible for activation. FALLING-BLOCK constraint can become a candidate for activation only if it is the case that $w \not \models \ll e x i s t s$, gravity, $0 \gg$, i.e., if the absence of gravity is not known in the background situation [2].

Forward-chaining mechanism of BABY-SIT is initiated either when the user tells the system to do so or by assertion of a new object into the system. A candidate forwardchaining constraint is activated whenever its antecedent part is satisfied. All the consequences are asserted if they do not yield a contradiction in the situation into which they are asserted. New assertions may in turn activate other candidate forward-chaining constraints. Candidate backward-chaining constraints are activated either when a query is entered explicitly or is issued by the forward-chaining mechanism. In BABY-SIT, constraints, as classified by Black in [15], between situation types as well as between infons of a situation can be easily modeled.

Query mode enables one to issue queries about situations. BABY-SIT's response depends on its understanding of the intention of the user. There are several possible actions which can be further controlled by the user:

- Searching for solutions by using a given group of constraints.

- Replacing each parameter in the query expression by the corresponding individual if there is a possible anchor, either partial or full, for that parameter provided by the given anchoring situation.

- Returning solutions. (Their number is determined by the user.)

- Displaying a solution with its parameters replaced by the individuals to which they are anchored by the given anchoring situation.

- For each solution, displaying infons anchoring any parameter in the solution to an individual in the given anchoring situation.

- Displaying a trace of anchoring of parameters in each solution.

The computation upon issuing a query is done either by direct querying through situations or by the application of backward-chaining constraints. ${ }^{23}$ A situation, s, supports an

\footnotetext{
${ }^{23}$ In addition to query operations, a special operation, oracle, is allowed in the query mode. An oracle is defined over an object and a set of infons (set of issues) [25]. The oracle of an object enables one to chronologically view the information about that object from a particular perspective provided by the given set of infons. One may consider oracles as 'histories' of specific objects. Given an object and a set of issues, BABY-SIT anchors all parameters in this set of issues and. collects all infons supported by the situations in the system under a specific situation, thus forming a 'minimal' situation which supports all parameter-free infons in the set of issues.
} 
infon if the infon is either explicitly asserted to hold in $s$, or it is supported by a situation $s^{\prime}$ which is part of $s$, or it can be proven to hold by application of backward-chaining constraints. Given an anchoring situation, say anchor1, a query and the system's response to it are as follows:

$\mathrm{Q}>$ ?S $\vDash\{\ll s e e, \mathrm{E}, ? \mathrm{Y}, 1 \gg, \ll$ time-of, sit1, ?Z, 1》\}, $w \not \mid \ll$ blind, bob, $1 \gg$

answers (without anchoring of parameters):

sit $3 \models\{\ll s e e, \mathrm{E}$, sit $1,1 \gg, \ll t i m e-o f$, sit $1, t 1,1 \gg\}$, $w \not=\ll b l i n d, b a b, 1 \gg$

with the anchoring on parameters:

anchor $1 \vDash \ll$ anchor, $\mathrm{E}$, bob, $1 \gg$.

BABY-SIT enhances the basic features of situation theory. Situations are viewed at an abstract level. This means that situations are sets of parametric infons, but they may be non-well-founded (circularity) $[1,10]$. Parameters are place holders and can be anchored to unique individuals in an anchoring situation. A situation can be realized if its parameters are anchored, either partially or fully, by an anchoring situation. That is, only anchoring the parameters of an infon contributes a piece of information about the situation. Each relation has 'appropriateness conditions' which determine the type of its arguments. Situations (and hence infons they support) can have spatio-temporal dimensions. A hierarchy of situations can be defined both statically and dynamically. A situation can have information about another which is part of the former. Situations and constraints can be grouped to form a whole which provides a computational context. Moreover, partial nature of situations facilitates computation with incomplete information.

Objects in the environment and the attainment of information flow are compatible with the ontology of situation theory. Computation is context-sensitive and type-theoretic. The mode of computation is built upon conveyance and inheritance of information, consistency of situations, and constraint satisfaction. ${ }^{24}$ Information inheritance supported by BABY-SIT, together with the information conveyance among situations, enables one to use contextual information which plays a critical role in all forms of behavior and communication. Building models of reasoning from various 'points of view' is possible, especially via grouping of constraints and anchoring situations.

\section{Concluding Remarks}

Serious thinking about the computational aspects of the situation theory is just starting. There have been only a few proposals $[17,33,40,46,52,59]$ in this direction, with varying degrees of divergence from the ontology of situation theory. ASTL [17] and PROSIT [52] mainly offer a Prolog- or Lisp-like programming language while BABY-SIT [59] provides a programming environment incorporating situationtheoretic constructs. These approaches are primarily motivated by situation theory and situation semantics, and can be employed as general programming and knowledge representation languages in various domains of application. Situation schemata [33], however, are theoretical tools built more for

\footnotetext{
${ }^{24} \mathrm{KEE}$ environment supports both a truth maintenance system built upon de Kleer's work on assumption-based truth maintenance [45] and a world system based on Morris and Nado's work [47]. Since these two systems are employed at its most primitive layer, BABY-SIT can be said to be complete as well as sound.
}

\begin{tabular}{|l|c|c|c|}
\hline Constraint Type & PROSIT & ASTL & BABY-SIT \\
\hline Nomic & $\sqrt{ }$ & $\sqrt{ }$ & $\sqrt{ }$ \\
\hline Necessary & $\sqrt{ }$ & $\sqrt{ }$ & $\sqrt{ }$ \\
\hline Conventional & - & - & $?$ \\
\hline Conditional & - & - & $\sqrt{ }$ \\
\hline Situated & $\sqrt{ }$ & - & $\sqrt{ }$ \\
\hline Global & - & $\sqrt{ }$ & $\sqrt{ }$ \\
\hline
\end{tabular}

\begin{tabular}{|l|c|c|c|}
\hline Constraint Class & PROSIT & ASTL & BABY-SIT \\
\hline Situation constraint & - & $\sqrt{ }$ & $\sqrt{ }$ \\
\hline Infon constraint & $\sqrt{ }$ & $\sqrt{ }$ & $\sqrt{ }$ \\
\hline Argument constraint & - & - & $\sqrt{ }$ \\
\hline
\end{tabular}

\begin{tabular}{|l|c|c|c|}
\hline Computation Mode & PROSIT & ASTL & BABY-SIT \\
\hline Unification & $\sqrt{ }$ & $\sqrt{ }$ & $\sqrt{ }$ \\
\hline Type-theoretic & - & - & $\sqrt{ }$ \\
\hline Coherence & - & - & $\sqrt{ }$ \\
\hline Forward-chaining & $\sqrt{ }$ & - & $\sqrt{ }$ \\
\hline Backward-chaining & $\sqrt{ }$ & $\sqrt{ }$ & $\sqrt{ }$ \\
\hline Bidirectional-chaining & $\sqrt{ }$ & - & $\sqrt{ }$ \\
\hline
\end{tabular}

\begin{tabular}{|l|c|c|c|}
\hline Miscellaneous Features & PROSIT & ASTL & BABY-SIT \\
\hline Circularity & $\sqrt{ }$ & $\sqrt{ }$ & $\sqrt{ }$ \\
\hline Partiality & $\sqrt{ }$ & $\sqrt{ }$ & $\sqrt{ }$ \\
\hline Parameters & $?$ & $?$ & $\sqrt{ }$ \\
\hline Abstraction & $?$ & $?$ & $\sqrt{ }$ \\
\hline Anchoring & $?$ & $?$ & $\sqrt{ }$ \\
\hline Information nesting & $\sqrt{ }$ & $\sqrt{ }$ & $\sqrt{ }$ \\
\hline Set operations & $\sqrt{ }$ & - & - \\
\hline Oracles & - & - & $?$ \\
\hline
\end{tabular}

Legend: $\sqrt{ }$ : exists, $-:$ doesn't exist, ?: partially/conceptually exists.

Table 1: Tableau comparison of existing approaches.

knowledge representation than programming, specifically tailored for semantic interpretation from linguistic form. EPILOG [40] differs from these approaches in that it is influenced by situation semantics rather than motivated directly by the situation theory and situation semantics' theoretical apparatus. Lespérance's work [46] is a theoretical attempt towards exploiting computational aspects of situation semantics.

PROSIT, ASTL, and BABY-SIT are specifically designed with mechanisms allowing state of the art constructs of situation theory. Though differing in detail (cf. Table 1), these approaches are useful initial attempts towards a computational account of the theory.

We believe that computational aspects of situation theory call for deeper investigation. Although the current attempts are in their infancy, they already warrant interest in domains of artificial intelligence and natural language processing. However, their use should be further demonstrated to prove why situation theory provides a challenging ground for solving various phenomena in these fields and possibly in others.

\section{Acknowledgments}

We gratefully acknowledge the thoughtful comments of an anonymous SIGART referee on a preliminary version of this paper. 


\section{Note}

Some of our manuscripts on situation theory are available in Postscript format via ftp: login anonymously to ftp.cs.bilkent.edu.tr and see under the directory /pub/tech-reports.

\section{References}

[1] P. Aczel. Non-Well-Founded Sets, CSLI Lecture Notes Number 14, Center for the Study of Language and Information, Stanford, CA, 1988.

[2] V. Akman and E. Tin. "What is in a Context?" in E. Arlkan, editor, Proceedings of the 1990 Bilkent International Conference on New Trends in Communication, Control, and Signal Processing, Volume 2, Amsterdam, Holland: Elsevier, 1990, pp. 1670-1676.

[3] N. Asher. "Verbal Information, Interpretation, and Attitudes" in P. P. Hanson, editor, Information, Language, and Cognition, Vancouver, Canada: The University of British Columbia Press, 1990, pp. 29-56.

[4] J. L. Austin. "Truth," in J. O. Urmson and G. J. Warnock, editors, Philosophical Papers of J. L. Austin, Oxford, U.K.: Oxford University Press, 1961, pp. 117133.

[5] J. Barwise. "An Introduction to First-Order Logic," in J. Barwise, editor, Handbook of Mathematical Logic, Amsterdam, Holland: North-Holland, 1977, pp. 5-46.

[6] J. Barwise. "Model-Theoretic Logics: Background and Aims," in J. Barwise and S. Feferman, editors, ModelTheoretic Logics, Berlin, Germany: Springer-Verlag, 1985, pp. 3-23.

[7] J. Barwise. "Noun Phrases, Generalized Quantifiers, and Anaphora," in P. Gärdenfors, editor, Generalized Quantifiers, Dordrecht, Holland: Reidel, 1987, pp. 1-29.

[8] J. Barwise. The Situation in Logic, CSLI Lecture Notes Number 17, Center for the Study of Language and Information, Stanford, CA, 1989.

[9] J. Barwise and R. Cooper. Extended Kamp Notation: A Graphical Notation for Situation Theory, Research Paper No. HCRC/RP-38, Human Communication Research Center, University of Edinburgh, Edinburgh, U.K., 1992 .

[10] J. Barwise and J. Etchemendy. The Liar: An Essay on Truth and Circularity, New York, N.Y.: Oxford University Press, 1987.

[11] J. Barwise and J. Etchemendy. "Model-Theoretic Semantics," in M. I. Posner, editor, Foundations of Cognitive Science, Cambridge, MA: MIT Press, 1989, pp. 207-243.

[12] J. Barwise and J. Perry. Situations and Attitudes, Cambridge, MA: MIT Press, 1983.

[13] J. Barwise and J. Perry. "Semantic Innocence and Uncompromising Situations," in A. P. Martinich, editor, The Philosophy of Language, New York, N.Y.: Oxford University Press, 1985, pp. 401-413.

[14] J. van Benthem. Essays in Logical Semantics, Dordrecht, Holland: Reidel, 1986.
[15] A. W. Black. "Constraints in Computational Situation Semantics," Lecture Notes circulated during the Logic, Language, and Information Summer School, Saarbrücken, Germany, 1991.

[16] A. W. Black. "Embedding DRT in a Situation-Theoretic Framework," in Proceedings of the Fifteenth International Conference on Computational Linguistics, Nantes, France, 1992, pp. 1116-1120.

[17] A. W. Black. A Situation Theoretic Approach to Computational Semantics, Ph.D. Thesis, Department of Artificial Intelligence, University of Edinburgh, Edinburgh, U.K., 1993.

[18] C. C. Chang and H. J. Keisler. Model Theory, Third Edition, Amsterdam, Holland: North-Holland, 1990.

[19] N. Chomsky. Lectures on Government and Binding, Dordrecht, Holland: Foris, 1981.

[20] E. Colban. "Prepositional Phrases in Situation Schemata," Appendix A in Fenstad et al., 1987.

[21] R. Cooper. "Tense and Discourse Location in Situation Semantics," Linguistics and Philosophy, 9: 17-36, 1986.

[22] R. Cooper. "Meaning Representation in Montague Grammar and Situation Semantics," in B. G. T. Lowden, editor, Proceedings of the Alvey Sponsored Workshop on Formal Semantics in Natural Language Processing, 1987.

[23] R. Cooper. "Three Lectures on Situation Theoretic Grammar," in M. Filgueiras, L. Damas, N. Moreira, and A. P. Tomás, editors, Natural Language Processing, Lecture Notes in Artificial Intelligence, Volume 476, Berlin, Germany: Springer-Verlag, 1991, pp. 102-140.

[24] R. Cooper, K. Mukai, and J. Perry, editors. Situation Theory and Its Applications, Volume 1, CSLI Lecture Notes Number 22, Center for the Study of Language and Information, Stanford, CA, 1990.

[25] K. Devlin. Logic and Information, Cambridge, U.K.: Cambridge University Press, 1991.

[26] K. Devlin. "Infons as Mathematical Objects," Minds and Machines, 2: 185-201, 1992.

[27] D. Dowty, R. Wall, and S. Peters. Introduction to Montague Semantics, Dordrecht, Holland: Reidel, 1981.

[28] F. Dretske. Seeing and Knowing, Chicago, IL: University of Chicago Press, 1969.

[29] F. Dretske. Knowledge and the Flow of Information, Cambridge, MA.: MIT Press, 1981.

[30] M. Ersan and V. Akman. Situated Modeling of Epistemic Puzzles, Technical Report No. BU-CEIS-94-17, Department of Computer Engineering and Information Science, Bilkent University, Ankara, Turkey, 1994.

[31] D. A. Evans. "A Situation Semantics Approach to the Analysis of Speech Acts," in Proceedings of the Nineteenth Annual Meeting of the Association for Computational Linguistics, 1981.

[32] G. Evans. The Varieties of Reference, New York, N.Y.: Oxford University Press, 1991. 
[33] J. E. Fenstad, P.-K. Halvorsen, T. Langholm, and J. van Benthem. Situations, Language, and Logic, Dordrecht, Holland: Reidel, 1987.

[34] J. E. Fenstad. "Natural Language Systems," in R. T. Nossum, editor, Advanced Topics in Artificial Intelligence: 2nd Advanced Course, Lecture Notes in Artificial Intelligence, Volume 345, Berlin, Germany: SpringerVerlag, 1987, pp. 189-233.

[35] J. M. Gawron and S. Peters. Anaphora and Quantification in Situation Semantics, CSLI Lecture Notes Number 19, Center for the Study of Language and Information, Stanford, CA, 1990.

[36] P. Geach. Reference and Generality, Ithaca, N.Y.: Cornell University Press, 1962.

[37] H. P. Grice. "Utterer's Meaning, Sentence-Meaning, and Word-Meaning," Foundations of Language, 4: 1-18, 1968.

[38] Z. S. Harris. A Theory of Language and Information: A Mathematical Approach, New York, N.Y.: Oxford University Press, 1991.

[39] J. R. Hobbs, M. E. Stickel, D. E. Appelt, and P. Martin. "Interpretation as Abduction," Artificial Intelligence, 63: $69-142,1993$.

[40] C. H. Hwang and L. K. Schubert. "Episodic Logic: A Situational Logic for Natural Language Processing," in P. Aczel, D. Israel, Y. Katagiri, and S. Peters, editors, Situation Theory and Its Applications, Volume 3, CSLI Lecture Notes Number 37, Center for the Study of Language and Information, Stanford, CA, 1993, pp. 303338 .

[41] D. Israel and J. Perry. "What is Information?" in P. P. Hanson, editor, Information, Language, and Cognition, Vancouver, Canada: The University of British Columbia Press, 1990, pp. 1-28.

[42] H. Kamp. "A Theory of Truth and Semantic Representation," in J. Groenendijk, T. Janssen, and M. Stokhof, editors, Formal Methods in the Study of Language, Amsterdam, Holland: Mathematical Center, 1981, pp. 277322 .

[43] H. Kamp and U. Reyle. From Discourse to Logic (Introduction to Model-Theoretic Semantics of Natural Language, Formal Logic, and Discourse Representation Theory), Parts I and II, Studies in Logic and Philosophy, Volume 42, Dordrecht, Holland: Kluwer, 1993.

[44] $K E E^{T M}$ (Knowledge Engineering Environment) Software Development System, Version 4.1, IntelliCorp, Inc., Mountain View, CA, 1993.

[45] J. de Kleer. "An Assumption-Based Truth Maintenance System," Artificial Intelligence, 28(2): 127-162, 1986.

[46] Y. Lespérance. "Toward a Computational Interpretation of Situation Semantics," Computational Intelligence, 2: 9-27, 1986.

[47] P. H. Morris and R. A. Nado. "Representing Actions with an Assumption-Based Truth Maintenance System:" in Proceedings of the Fifth International Conference on Artificial Intelligence, Philadelphia, PA, 1986, pp. 1317.
[48] H. Nakashima, S. Peters, and H. Schütze. "Communication and Inference through Situations," in Proceedings of the Third Conference on Artificial Intelligence Applications, Washington, D.C.: IEEE Computer Society Press, 1987, pp. 76-81.

[49] H. Nakashima, H. Suzuki, P.-K. Halvorsen, and S. Peters. "Towards a Computational Interpretation of Situation Theory," in Proceedings of the International Conference on Fifth Generation Computer Systems, Institute for New Generation Computer Technology, Tokyo, Japan, 1988, pp. 489-498.

[50] J. Perry. "Contradictory Situations," in F. Landman and F. Veltman, editors, Varieties of Formal Semantics, Dordrecht, Holland: Foris, 1984, pp. 313-324.

[51] M. Rooth. Noun Phrase Interpretation in Montague Grammar, File Change Semantics, and Situation Semantics, Report No. CSLI-86-51, Center for the Study of Language and Information, Stanford, CA, 1986.

[52] H. Schütze. "The PROSIT Language v0.4," Manuscript, Center for the Study of Language and Information, Stanford University, Stanford, CA, 1991.

[53] J. R. Searle. Speech Acts: An Essay in the Philosophy of Language, Cambridge, U.K.: Cambridge University Press, 1969.

[54] P. Sells. Lectures on Contemporary Syntactic Theories, CSLI Lecture Notes Number 3, Center for the Study of Language and Information, Stanford, CA, 1985.

[55] D. Sperber and D. Wilson. Relevance: Communication and Cognition, Oxford, U.K.: Basil Blackwell, 1986.

[56] G. L. Steele, Jr. Common Lisp: The Language, Second Edition, Bedford, MA: Digital Press, 1990.

[57] L. Sterling and E. Shapiro. The Art of Prolog: Advanced Programming Techniques, Cambridge, MA: MIT Press, 1986.

[58] S. Stucky. "The Situated Processing of Situated Language," Linguistics and Philosophy, 12: 347-357, 1989.

[59] E. Tin and V. Akman. "BABY-SIT: A Computational Medium Based on Situations," in Proceedings of the 9th Amsterdam Colloquium, ILLC/Department of Philosophy, University of Amsterdam, Amsterdam, The Netherlands, 1993 (to appear).

[60] E. Tin and V. Akman. "BABY-SIT: Towards a Situation-Theoretic Computational Environment," in C. Martín-Vide, editor, Current Issues in Mathematical Linguistics, Amsterdam, Holland: Elsevier, 1994 (to appear).

[61] E. Tin ánd V. Akman. "Information-Oriented Computation with BABY-SIT," in Conference on InformationOriented Approaches to Logic, Language, and Computation (4th Conference on Situation Theory and its Applications), Saint Mary's College of California, Moraga, CA, 1994 (accepted). 